DOI 10.4171/JEMS/240

Daniel Huybrechts

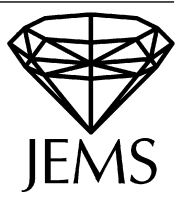

\title{
Chow groups of K3 surfaces and spherical objects
}

Received October 27, 2008 and in revised form February 2, 2009

Abstract. We show that for a $\mathrm{K} 3$ surface $X$ the finitely generated subring $R(X) \subset \mathrm{CH}^{*}(X)$ introduced by Beauville and Voisin is preserved under derived equivalences. This is proved by analyzing Chern characters of spherical bundles (and complexes). As for a K3 surface $X$ defined over a number field all spherical bundles on the complex K3 surface $X_{\mathbb{C}}$ are defined over $\overline{\mathbb{Q}}$, this is compatible with the Bloch-Beilinson conjecture. Besides the work of Beauville and Voisin [5], Lazarfeld's result on Brill-Noether theory for curves in K3 surfaces $[15]$ and the deformation theory developed in [12] are central for the discussion.

\section{Introduction}

The Chow group $\mathrm{CH}^{i}(X)$ of a smooth projective variety $X$ is the group of all cycles of codimension $i$ modulo rational equivalence (see [8]). For surfaces these are $\mathrm{CH}^{0}(X)=$ $\mathbb{Z}[X], \mathrm{CH}^{1}(X) \simeq \operatorname{Pic}(X)$ (via the first Chern class), and the more mysterious $\mathrm{CH}^{2}(X)$. The latter is roughly the group of 0 -cycles $Z=\sum n_{i} x_{i}$ modulo linear equivalence on curves containing $Z$. Since Mumford's article [20] one knows that, unlike $\mathrm{CH}^{0}(X)$ and $\mathrm{CH}^{1}(X)$, the group $\mathrm{CH}^{2}(X)$ can be big. More precisely, the subgroup $A(X):=$ $\operatorname{ker}\left(\operatorname{deg}: \mathrm{CH}^{2}(X) \rightarrow \mathbb{Z}\right)$ of all homologically trivial 0 -cycles on a complex projective surface $X$ is infinite-dimensional (and in particular infinitely generated) whenever $p_{g}(X)=h^{2}\left(X, \mathcal{O}_{X}\right)>0$, e.g. for $\mathrm{K} 3$ surfaces.

For K3 surfaces, Beauville and Voisin have studied more recently the subgroup

$$
R(X):=\mathrm{CH}^{0}(X) \oplus \mathrm{CH}^{1}(X) \oplus c_{X} \mathbb{Z} \subset \mathrm{CH}^{2}(X),
$$

where $c_{X} \in \mathrm{CH}^{2}(X)$ is the fundamental class of a closed point $x \in X$ that is contained in a (possibly) singular rational curve in $X$. As shown in [5], the class $c_{X}$ is independent of the point $x$. The main results of [5] can be stated as follows:

Theorem (Beauville-Voisin).

(i) $R(X) \subset \mathrm{CH}^{*}(X)$ is a subring.

(ii) $24 c_{X}=c_{2}(X)$.

D. Huybrechts: Mathematisches Institut, Universität Bonn, Beringstr. 1, 53115 Bonn, Germany; e-mail: huybrech@math.uni-bonn.de 
The first condition is equivalently expressed by saying that for any line bundle $L \in \operatorname{Pic}(X)$ the class $\mathrm{c}_{1}(L)^{2} \in \mathrm{CH}^{2}(X)$ is a multiple of $c_{X}$.

Let us rephrase (i) and (ii) in terms of Mukai vectors. For any coherent sheaf (or complex of coherent sheaves) $E$ on $X$ one defines

$$
v^{\mathrm{CH}}(E):=\operatorname{ch}(E) \sqrt{\operatorname{td}(X)} \in \mathrm{CH}^{*}(X) .
$$

In other words, $v^{\mathrm{CH}}(X):=\left(\operatorname{rk}(E), \mathrm{c}_{1}(E), \operatorname{ch}_{2}(E)+\operatorname{rk}(E) c_{X}\right) \in \mathrm{CH}^{0}(X) \oplus \mathrm{CH}^{1}(X) \oplus$ $\mathrm{CH}^{2}(X)$. Then by applying (i) to powers of $L$, conditions (i) and (ii) can be reformulated as:

(iii) For any line bundle $L \in \operatorname{Pic}(X)$, one has $v^{\mathrm{CH}}(L) \in R(X)$.

Line bundles on a K3 surface $X$ are the easiest examples of spherical objects on $X$, which by definition are bounded complexes of coherent sheaves $E \in \mathrm{D}^{\mathrm{b}}(X)$ with $\operatorname{Ext}_{X}^{*}(E, E) \simeq H^{*}\left(\mathrm{~S}^{2}, \mathbb{C}\right)$. Building upon [5], we shall prove the following generalization of (iii).

Theorem 1. Let $X$ be a complex projective K3 surface of Picard number $\rho(X) \geq 2$ and let $E \in \mathrm{D}^{\mathrm{b}}(X)$ be a spherical object. Then $v^{\mathrm{CH}}(E) \in R(X)$.

Spherical objects play a distinguished role in the study of the bounded derived category $\mathrm{D}^{\mathrm{b}}(X)$ (and its homological mirror given by a certain Fukaya category). They are essential for the understanding of the rich structure of the group $\operatorname{Aut}\left(\mathrm{D}^{\mathrm{b}}(X)\right)$ of all exact $\mathbb{C}$-linear autoequivalences and Bridgeland's space of stability conditions $\operatorname{Stab}(X)$ (see [7]).

In this context, Theorem 1 is used to deduce information about the action of derived equivalences on the level of Chow groups. More precisely, we have

Theorem 2. Let $\Phi_{\mathcal{E}}: \mathrm{D}^{\mathrm{b}}(X) \stackrel{\sim}{\rightarrow} \mathrm{D}^{\mathrm{b}}\left(X^{\prime}\right)$ be an exact $\mathbb{C}$-linear equivalence between the bounded derived categories of two smooth complex projective K3 surfaces of Picard number $\rho(X) \geq 2$. Then the induced action $\Phi_{\mathcal{E}}^{\mathrm{CH}}: \mathrm{CH}^{*}(X) \stackrel{\sim}{\rightarrow} \mathrm{CH}^{*}\left(X^{\prime}\right)$ preserves the Beauville-Voisin ring, i.e.

$$
\Phi_{\mathcal{E}}^{\mathrm{CH}}(R(X))=R\left(X^{\prime}\right)
$$

The key step towards Theorem 1 is the following result which is valid without any assumption on the Picard group of the surface (see Theorem 2.3.

Theorem 3. Let $X$ and $X^{\prime}$ be complex projective $K 3$ surfaces and

$$
\Phi_{\mathcal{E}}, \Phi_{\mathcal{F}}: \mathrm{D}^{\mathrm{b}}(X) \stackrel{\sim}{\rightarrow} \mathrm{D}^{\mathrm{b}}\left(X^{\prime}\right)
$$

be two Fourier-Mukai equivalences. If their induced actions on cohomology coincide, i.e. $\Phi_{\mathcal{E}}^{H}=\Phi_{\mathcal{F}}^{H}: \widetilde{H}(X, \mathbb{Z}) \stackrel{\sim}{\rightarrow} \widetilde{H}\left(X^{\prime}, \mathbb{Z}\right)$, then also $\Phi_{\mathcal{E}}^{\mathrm{CH}}=\Phi_{\mathcal{F}}^{\mathrm{CH}}: \mathrm{CH}(X) \stackrel{\sim}{\rightarrow} \mathrm{CH}\left(X^{\prime}\right)$.

In particular, for $X=X^{\prime}$ the result shows that $\Phi_{\mathcal{E}}^{\mathrm{CH}}$ acts as the identity on $A(X)$ whenever it acts trivially on cohomology (see Corollary 2.7). As we will explain in Remark 2.9. this is predicted by a general conjecture of Bloch which asserts that the action of any 
algebraic correspondence on the graded pieces of his conjectural filtration is determined by its action on cohomology (see [3, 1.8] or [24, Conj. 23.22]).

If instead of projective $\mathrm{K} 3$ surfaces over $\mathbb{C}$ we consider $\mathrm{K} 3$ surfaces $X$ defined over a number field $K$, then the situation changes completely. In this case $\mathrm{CH}^{2}(X)$ is no longer expected to be infinitely generated. In fact, the Bloch-Beilinson conjectures predict that for $\mathrm{K} 3$ surfaces over number fields the degree map yields an isomorphism $\mathrm{CH}^{2}(X) \otimes \mathbb{Q}$ $\simeq \mathbb{Q}$ (see [4, 21]). This can be rephrased as the following

Conjecture (Bloch-Beilinson for K3 surfaces). For any K3 surface $X$ defined over a number field $K$ base change yields

$$
\mathrm{CH}^{*}(X) \otimes \mathbb{Q} \hookrightarrow \mathrm{CH}^{*}\left(X_{L}\right) \otimes \mathbb{Q} \stackrel{\sim}{\rightarrow} R\left(X_{\mathbb{C}}\right) \otimes \mathbb{Q} \simeq \mathbb{Q}^{\rho\left(X_{\mathbb{C}}\right)+2},
$$

where $L / K$ is a certain finite field extension with a chosen embedding $L \subset \mathbb{C}$.

As usual, $X_{L}$ denotes $X \times_{\operatorname{Spec}(K)} \operatorname{Spec}(L)$, and similarly for $X_{\mathbb{C}}$, which is then a complex projective $\mathrm{K} 3$ surface.

It is well known that the base change $\mathrm{CH}^{*}(X) \rightarrow \mathrm{CH}^{*}\left(X_{L}\right)$ for any extension $L / K$ has torsion kernel. The passage to the finite extension $L / K$ is not essential and only needed to ensure that all geometric line bundles are defined. Thus, the central point of the conjecture is that $\mathrm{CH}^{2}(X) \otimes \mathbb{Q} \simeq \mathbb{Q} c_{X}$. For a proof it would clearly suffice to prove that any rational point $x \in X(\overline{\mathbb{Q}})$ satisfies $[x]=c_{X}$, but there is no obvious geometric reason for this.

The skyscraper sheaves $k(x)$ of rational points $x \in X(\overline{\mathbb{Q}})$ define semi-rigid objects in $\mathrm{D}^{\mathrm{b}}\left(X_{\overline{\mathbb{Q}}}\right)$ (see Section 5 for the definition), for $\operatorname{Ext}^{1}(k(x), k(x))$ is two-dimensional. In this sense, they are reasonably close to our spherical objects $E \in \mathrm{D}^{\mathrm{b}}(X)$ which have vanishing $\operatorname{Ext}^{1}(E, E)$. The following is thus in accordance with the Bloch-Beilinson conjecture for K3 surfaces.

Theorem 3. Let $X$ be a $K 3$ surface over a number field $K$. Then

- Any spherical object $E \in \mathrm{D}^{\mathrm{b}}\left(X_{\mathbb{C}}\right)$ is defined over some finite extension $L / K$.

- Any spherical object $F \in \mathrm{D}^{\mathrm{b}}\left(X_{\overline{\mathbb{Q}}}\right)$ satisfies $v^{\mathrm{CH}}\left(F_{\mathbb{C}}\right) \in R\left(X_{\mathbb{C}}\right)$ if in addition $\rho\left(X_{\overline{\mathbb{Q}}}\right)$ $\geq 2$.

Together these two assertions show that on any K3 surface $X$ defined over $\overline{\mathbb{Q}}$ with Picard number $\rho(X) \geq 2$ there exist a large number of non-trivial classes in $\mathrm{CH}^{2}(X)$ for which the Bloch-Beilinson conjecture can be verified. As with other approaches to the conjecture, the difficult part, that would show that this suffices to deduce the result for all classes, remains open.

Despite the algebraic nature of all assertions, non-algebraic K3 surfaces play a crucial but hidden role in this paper. A technique that has been developed together with Macrì and Stellari in [12] allows one to deform any derived equivalence that acts as the identity on cohomology to an essentially trivial derived equivalence on a generic and non-algebraic deformation of $X$. This is explained in Section 2. The results can also be used to show that the natural representations of $\operatorname{Aut}\left(\mathrm{D}^{\mathrm{b}}(X)\right)$ on $\mathrm{CH}^{*}(X)$ and on the Mukai lattice $\widetilde{H}(X, \mathbb{Z})$ encode the same information (see Corollary 2.7). 
In Section 3 we reduce Theorem 1 to the case that $E$ is a spherical vector bundle. This section also explains how Theorem 2 is deduced from Theorem 1 . The case of spherical vector bundles is dealt with in detail in Section 4. The main ingredient here is Lazarsfeld's result that the generic curve in an indecomposable linear system on a K3 surface is BrillNoether general [15]. The final Section 5 discusses the relation to the Bloch-Beilinson conjecture for K3 surfaces over number fields.

We certainly expect all results to hold true without any assumption on the Picard number of the K3 surface $X$ and, in fact, Theorem 1 can be proved also for $\rho(X)=1$ under additional numerical conditions on the spherical object $E$.

Notation. By $\mathrm{D}^{\mathrm{b}}(X)$ we denote the bounded derived category of the abelian category $\operatorname{Coh}(X)$ of coherent sheaves on $X$. It will be considered as a $K$-linear triangulated category, when $X$ is defined over $K$ (which mostly is $\mathbb{C}, \overline{\mathbb{Q}}$ or a number field). The Mukai lattice $\widetilde{H}(X, \mathbb{Z})$ of a complex $\mathrm{K} 3$ surface is by definition the full singular cohomology $H^{*}(X, \mathbb{Z})$ endowed with its natural weight two Hodge structure and the Mukai pairing (see e.g. [10]). All intersection products will be taken with respect to the Mukai pairing which differs from the usual intersection pairing by a sign in degree four. We will associate to any $E \in \mathrm{D}^{\mathrm{b}}(X)$ its Mukai vector $v(E):=\operatorname{ch}(E) \sqrt{\operatorname{td}(X)} \in \widetilde{H}(X, \mathbb{Z})$ and its natural lift to $\mathrm{CH}^{*}(X)$, which is denoted $v^{\mathrm{CH}}(E)$. We do not make this distinction for the characteristic classes.

\section{Fourier-Mukai action on the Chow group}

Let us start by briefly recalling the following examples of Fourier-Mukai equivalences for K3 surfaces.

(i) For a line bundle $L \in \operatorname{Pic}(X)$, the tensor product $L \otimes($ ) defines a Fourier-Mukai equivalence

$$
\Phi_{\iota_{*} L}: \mathrm{D}^{\mathrm{b}}(X) \stackrel{\sim}{\rightarrow} \mathrm{D}^{\mathrm{b}}(X)
$$

where $\iota_{*} L$ is the direct image of $L$ under the diagonal embedding $X \stackrel{\sim}{\rightarrow} \Delta \subset X \times X$.

(ii) If $X^{\prime}$ is a smooth projective two-dimensional fine moduli space of $\mu$-stable vector bundles on $X$, then the universal bundle $\mathbb{E}$ on $X \times X^{\prime}$ induces an equivalence (see [19, 10])

$$
\Phi_{\mathbb{E}}: \mathrm{D}^{\mathrm{b}}(X) \stackrel{\sim}{\rightarrow} \mathrm{D}^{\mathrm{b}}\left(X^{\prime}\right) .
$$

Note that any Fourier-Mukai partner of $X$ is isomorphic to such a moduli space, but of course other Fourier-Mukai equivalences between $X$ and $X^{\prime}$ do exist and are given by kernels more complicated than $\mathbb{E}$.

(iii) If $E \in \mathrm{D}^{\mathrm{b}}(X)$ is a spherical object, i.e. $\operatorname{Ext}_{X}^{*}(E, E) \simeq H^{*}\left(\mathrm{~S}^{2}, \mathbb{C}\right)$, then the spherical twist

$$
T_{E}: \mathrm{D}^{\mathrm{b}}(X) \stackrel{\sim}{\rightarrow} \mathrm{D}^{\mathrm{b}}(X),
$$

studied in detail in [23], is a Fourier-Mukai equivalence with kernel $\mathcal{P}_{E}:=\operatorname{Cone}(\operatorname{tr}$ : $E^{\vee} \otimes E \rightarrow \mathcal{O}_{\Delta}$ ) (see also [10, Ch. 8]). Spherical objects, although rigid, exist in 
abundance on any projective K3 surface. E.g. any line bundle, even the trivial one, gives rise to a non-trivial spherical twist. Moreover, Kuleshov shows in [14] that any $(1,1)$-class $v \in \widetilde{H}(X, \mathbb{Z})$ of square -2 is the Mukai vector of a spherical object, which can be chosen to be a vector bundle if the rank of $v$ is positive.

Any Fourier-Mukai equivalence $\Phi_{\mathcal{E}}: \mathrm{D}^{\mathrm{b}}(X) \stackrel{\sim}{\rightarrow} \mathrm{D}^{\mathrm{b}}\left(X^{\prime}\right)$ induces a group isomorphism

$$
\Phi_{\mathcal{E}}^{\mathrm{CH}}: \mathrm{CH}^{*}(X) \stackrel{\sim}{\rightarrow} \mathrm{CH}^{*}\left(X^{\prime}\right)
$$

and a Hodge isometry

$$
\Phi_{\mathcal{E}}^{H}: \widetilde{H}(X, \mathbb{Z}) \stackrel{\sim}{\rightarrow} \widetilde{H}\left(X^{\prime}, \mathbb{Z}\right) .
$$

Both are defined as correspondences associated to $v^{\mathrm{CH}}(\mathcal{E}) \in \mathrm{CH}^{*}\left(X \times X^{\prime}\right) \otimes \mathbb{Q}$ respectively $v(\mathcal{E}) \in H^{*}\left(X \times X^{\prime}, \mathbb{Q}\right)$.

Note that in general one would expect $\Phi^{\mathrm{CH}}$ and $\Phi^{H}$ to be defined only with rational coefficients, but as Mukai observed the situation is special for $\mathrm{K} 3$ surfaces (see the original argument in [19] or [10, 11]).

Remark 2.1. The action $\Phi_{\mathcal{E}}^{\mathrm{CH}}$ is difficult to grasp for example (ii), but easy to describe in examples (i) and (iii).

Indeed, in (i) the actions $\Phi_{\iota_{*} L}^{\mathrm{CH}}$ and $\Phi_{\iota_{*} L}^{H}$ are both given by multiplication with $\operatorname{ch}(L)=$ $\exp \left(\mathrm{c}_{1}(L)\right)$, where the Chern character is viewed in $\mathrm{CH}^{*}(X)$ resp. $H^{*}(X, \mathbb{Z})$. Thus Theorem 2 is a trivial consequence of the results in [5] in this case.

For the spherical twists in (iii), $T_{E}^{\mathrm{CH}}$ and $T_{E}^{H}$ are reflections in $v^{\mathrm{CH}}(E)^{\perp}$ resp. $v(E)^{\perp}$, where the orthogonal complement is taken with respect to the Mukai pairing. In particular, their squares $\left(T_{E}^{2}\right)^{\mathrm{CH}}$ and $\left(T_{E}^{2}\right)^{H}$ act trivially, i.e. as the identity, on both groups $\mathrm{CH}^{*}(X)$ resp. $\widetilde{H}(X, \mathbb{Z})$.

Remark 2.2. Observe that for an arbitrary spherical object the associated spherical twist $T_{E}$ preserves the Beauville-Voisin subring $R(X)$ if and only if $v^{\mathrm{CH}}(E) \in R(X)$. In this case it acts as the identity on the space of cohomologically trivial cycles $A(X)$.

According to Mumford, $\mathrm{CH}^{2}(X)$ is big and in fact of infinite dimension for any complex projective surface with $p_{g}(X)>0$ and therefore in particular for K3 surfaces. See [24, Ch. 22] for the notion of dimension of $\mathrm{CH}^{2}(X)$.

Thus, a priori for an arbitrary Fourier-Mukai equivalence $\Phi_{\mathcal{E}}: \mathrm{D}^{\mathrm{b}}(X) \stackrel{\sim}{\rightarrow} \mathrm{D}^{\mathrm{b}}\left(X^{\prime}\right)$ between two K3 surfaces the induced map $\Phi_{\mathcal{E}}^{\mathrm{CH}}: \mathrm{CH}^{*}(X) \stackrel{\sim}{\rightarrow} \mathrm{CH}^{*}\left(X^{\prime}\right)$ between the infinite dimensional Chow groups might capture more information than $\Phi_{\mathcal{E}}^{H}: \widetilde{H}(X, \mathbb{Z}) \stackrel{\sim}{\rightarrow}$ $\widetilde{H}\left(X^{\prime}, \mathbb{Z}\right)$. That this is (unfortunately?) not the case is the main result of this section:

Theorem 2.3. Let $X$ and $X^{\prime}$ be smooth complex projective K3 surfaces and let

$$
\Phi_{\mathcal{E}}, \Phi_{\mathcal{F}}: \mathrm{D}^{\mathrm{b}}(X) \stackrel{\sim}{\rightarrow} \mathrm{D}^{\mathrm{b}}\left(X^{\prime}\right)
$$

be two Fourier-Mukai equivalences with $\Phi_{\mathcal{E}}^{H}=\Phi_{\mathcal{F}}^{H}$. Then also

$$
\Phi_{\mathcal{E}}^{\mathrm{CH}}=\Phi_{\mathcal{F}}^{\mathrm{CH}}: \mathrm{CH}^{*}(X) \stackrel{\sim}{\rightarrow} \mathrm{CH}^{*}\left(X^{\prime}\right) .
$$


Remark 2.4. In general the direct sum decomposition $\mathrm{CH}^{*}(X)=\mathrm{CH}^{0}(X) \oplus \mathrm{CH}^{1}(X) \oplus$ $\mathrm{CH}^{2}(X)$ is not respected by Fourier-Mukai transforms. However, the homologically trivial part is. More precisely, if $\Phi_{\mathcal{E}}: \mathrm{D}^{\mathrm{b}}(X) \stackrel{\sim}{\rightarrow} \mathrm{D}^{\mathrm{b}}\left(X^{\prime}\right)$ is any Fourier-Mukai equivalence, then $\Phi_{\mathcal{E}}^{\mathrm{CH}}(A(X))=A\left(X^{\prime}\right)$. Moreover, if $\Phi_{\mathcal{E}}^{H}$ respects the cohomological degree, e.g. for cohomologically trivial autoequivalences, then $\Phi_{\mathcal{E}}^{\mathrm{CH}}\left(\mathrm{CH}^{0}(X) \oplus \mathrm{CH}^{2}(X)\right)=$ $\mathrm{CH}^{0}\left(X^{\prime}\right) \oplus \mathrm{CH}^{2}\left(X^{\prime}\right)$.

The essential step in the proof of Theorem 2.3 is the following slightly weaker result.

Proposition 2.5. Let $\Phi_{\mathcal{E}}, \Phi_{\mathcal{F}}: \mathrm{D}^{\mathrm{b}}(X) \stackrel{\sim}{\rightarrow} \mathrm{D}^{\mathrm{b}}\left(X^{\prime}\right)$ be as in Theorem 2.3. Then $\Phi_{\mathcal{E}}^{\mathrm{CH}}=$ $\Phi_{\mathcal{F}}^{\mathrm{CH}}$ on $\mathrm{CH}^{0}(X) \oplus \mathrm{CH}^{2}(X)$.

Proof. By studying the composition $\Phi_{\mathcal{F}}^{-1} \circ \Phi_{\mathcal{E}}: \mathrm{D}^{\mathrm{b}}(X) \stackrel{\sim}{\rightarrow} \mathrm{D}^{\mathrm{b}}(X)$, one easily reduces to the case of autoequivalences acting as the identity on cohomology. So let $\Phi_{\mathcal{E}_{0}}: \mathrm{D}^{\mathrm{b}}(X) \stackrel{\sim}{\rightarrow}$ $\mathrm{D}^{\mathrm{b}}(X)$ with $\Phi_{\mathcal{E}_{0}}^{H}=$ id. We claim that then also $\Phi_{\mathcal{E}_{0}}^{\mathrm{CH}}=$ id on $\mathrm{CH}^{0}(X) \oplus \mathrm{CH}^{2}(X)$. In particular we have to show that $\Phi_{\mathcal{E}_{0}}^{\mathrm{CH}}=$ id on the space of homologically trivial cycles $A(X)$.

Clearly, changing $\Phi_{\mathcal{E}_{0}}$ by even powers $T^{2 k}$ of the shift functor or even powers $T_{\mathcal{O}_{X}}^{2 k}$ of the spherical twist associated to the trivial line bundle does not affect the assertion (see Remark 2.2 and use $\left.v^{\mathrm{CH}}\left(\mathcal{O}_{X}\right)=\left(1,0, c_{X}\right) \in R(X)\right)$. So, in the course of the proof we will freely modify $\Phi_{\mathcal{E}_{0}}$ by autoequivalences of this type.

In [12] we were mainly interested in the case $\Phi_{\mathcal{E}_{0}}^{H}=\left(-\mathrm{id}_{H^{2}}\right) \oplus \mathrm{id}_{H^{0} \oplus H^{4}}$, but as mentioned there already the case $\Phi_{\mathcal{E}_{0}}^{H}=$ id is similar and actually easier. So the results of [12] show that for any autoequivalence $\Phi_{\mathcal{E}_{0}}$ with $\Phi_{\mathcal{E}_{0}}^{H}=$ id one finds:

(i) Two smooth formal deformations $\mathcal{X} \rightarrow \operatorname{Spf}(R) \leftarrow \mathcal{X}^{\prime}$ with $R=\mathbb{C}[[t]]$ and $\mathcal{X}_{0}=$ $X=\mathcal{X}_{0}^{\prime}$. Here $\mathcal{X}$ is the formal neighbourhood of $X$ inside its twistor space with respect to a very general Kähler class in $\operatorname{Pic}(X) \otimes \mathbb{R}$. Note that in this way $X$ is deformed towards a non-projective $\mathrm{K} 3$ surface.

(ii) A complex $\mathcal{E} \in \mathrm{D}^{\mathrm{b}}\left(\mathcal{X} \times_{R} \mathcal{X}^{\prime}\right):=\mathrm{D}_{\text {coh }}^{\mathrm{b}}\left(\mathcal{O}_{\mathcal{X} \times R} \mathcal{X}^{\prime-M o d}\right)$ deforming $\mathcal{E}_{0}$, i.e. $L \iota^{*} \mathcal{E} \simeq$ $\mathcal{E}_{0}$, where $\iota: X \times X \hookrightarrow \mathcal{X} \times{ }_{R} \mathcal{X}^{\prime}$ is the obvious closed embedding.

By [12, Prop. 2.18, 2.19] we may assume, after possibly composing with powers of $T_{\mathcal{O}}^{2}$ and $T^{2}$, that the restriction $\mathcal{E}_{K} \in \mathrm{D}^{\mathrm{b}}\left(\left(\mathcal{X} \times{ }_{R} \mathcal{X}^{\prime}\right)_{K}\right)$ of $\mathcal{E}$ to the general fibre is a sheaf. Hence [12, Cor. 4.5] applies and shows that there exists an $R$-flat sheaf (!) $\widetilde{\mathcal{E}}$ on $\mathcal{X} \times{ }_{R} \mathcal{X}^{\prime}$ with the same restriction to the general fibre as $\mathcal{E}$, i.e. $\widetilde{\mathcal{E}}_{K} \simeq \mathcal{E}_{K}$ in $\mathrm{D}^{\mathrm{b}}\left(\left(\mathcal{X} \times_{R} \mathcal{X}^{\prime}\right)_{K}\right)$, where $K=\mathbb{C}((t))$. For the notation we refer to [12]. Using the compatibilities between $\Phi_{\mathcal{E}_{0}}^{H}$ and the induced action on Hochschild (co)homology, one can in addition assume that the first order deformations $\mathcal{X}_{1} \rightarrow \operatorname{Spec}\left(\mathbb{C}[t] / t^{2}\right) \leftarrow \mathcal{X}_{1}^{\prime}$ of $X=\mathcal{X}_{0}=\mathcal{X}_{0}^{\prime}$ coincide.

The specialization morphism $K\left(\mathrm{D}^{\mathrm{b}}\left(\left(\mathcal{X} \times{ }_{R} \mathcal{X}^{\prime}\right)_{K}\right)\right) \rightarrow K\left(\mathrm{D}^{\mathrm{b}}(X \times X)\right)$ is well defined (see [12, Remark 2.7] or the analogous statement for Chow groups in Remark 4.5). Hence the coherent sheaf (!) $\widetilde{\mathcal{E}}_{0}$ and the original complex $\mathcal{E}_{0}$ have the same Mukai vectors $v^{\mathrm{CH}} \in$ $\mathrm{CH}^{*}(X \times X) \otimes \mathbb{Q}$ and therefore $\Phi_{\widetilde{\mathcal{E}}_{0}}^{\mathrm{CH}}=\Phi_{\mathcal{E}_{0}}^{\mathrm{CH}}$ and $\Phi_{\widetilde{\mathcal{E}}_{0}}^{H}=\Phi_{\mathcal{E}_{0}}^{H}$. 
Note that the Fourier-Mukai transform $\Phi_{G}$ associated to the sheaf $G:=\widetilde{\mathcal{E}}_{0}$ is not necessarily an equivalence, which would simplify the following arguments. But in any case, there is a dense open subset $U \subset X$ over which $G$ is flat (see e.g. [11, Thm. 2.15, Lemma 2.1.6]). Hence, for any closed point $x \in U$ the image $\Phi_{G}(k(x))$ is simply the sheaf $\left.G\right|_{\{x\} \times X}$. On the other hand, $v\left(\Phi_{G}(k(x))\right)=v\left(\Phi_{\mathcal{E}_{0}}(k(x))\right)=(0,0,1)$ and hence $\left.G\right|_{\{x\} \times X}$ must be of the form $k(y)$ for some point $y \in X$. This gives rise to a morphism $U \rightarrow X$, which by interchanging the two factors turns out to define a birational map $X \rightarrow X$. As any birational map between $\mathrm{K} 3$ surfaces, the latter can then be completed to an isomorphism $f: X \stackrel{\sim}{\rightarrow} X$. Moreover, if $Z:=\operatorname{Supp}(G) \subset X \times X$, then $\Gamma_{f} \subset Z$ is one irreducible component and the other components do not dominate $X$. The latter implies that $\left.\left[\Gamma_{f}\right]_{*}\right|_{H^{2,0}}=\left.\Phi_{G}^{H}\right|_{H^{2,0}}=\mathrm{id}_{H^{2,0}}$, i.e. $f$ is a symplectomorphism.

Obviously $f_{*}\left(c_{X}\right)=c_{X}$ and a general conjecture of Bloch (see Remark 2.9) predicts that for a symplectomorphism the induced automorphism $f_{*}: \mathrm{CH}^{*}(X) \simeq \mathrm{CH}^{*}(X)$ is the identity on $A(X)$. Since for generic $x \in X$ we have $\Phi_{G}^{\mathrm{CH}}([x])=[f(x)]$, this would be enough to conclude that $\Phi_{G}^{\mathrm{CH}}$ acts as the identity on $\mathrm{CH}^{2}(X)$.

Without using Bloch's conjecture, the argument is more involved and goes as follows. Since $G$ is the restriction of a sheaf on $\mathcal{X}_{1} \times R_{1} \mathcal{X}_{1}$, the structure sheaf of the graph $\mathcal{O}_{\Gamma_{f}}$ deforms sideways to first order, i.e. there exists an $R_{1}$-flat coherent sheaf on $\mathcal{X}_{1} \times{ }_{R_{1}} \mathcal{X}_{1}^{\prime}$ restricting to $\mathcal{O}_{\Gamma_{f}}$ over the closed point. (Do it first for the graph of $\left.f\right|_{U}$ and then pass to the closure.) In other words, the automorphism $f$ deforms sideways to first order (actually to any order, but we do not need this) in $\mathcal{X} \times{ }_{R} \mathcal{X}^{\prime}$. But clearly $f$ deforms sideways to first order if and only if $f^{*}(w)=w$, where $w \in H^{1}\left(X, \mathcal{T}_{X}\right)$ corresponds to the first order deformation $\mathcal{X}_{1} \rightarrow \operatorname{Spec}\left(\mathbb{C}[t] / t^{2}\right)$.

By construction, the class $w$ maps to the chosen Kähler class in $H^{1,1}(X)$ under the isomorphism $H^{1,1}(X) \simeq H^{1}\left(X, \Omega_{X}\right) \simeq H^{1}\left(X, \mathcal{T}_{X}\right)$ and, since the Kähler class was chosen generically, this implies $f^{*}=\mathrm{id}$ on $\operatorname{Pic}(X)$. Since the transcendental lattice $T(X)$ is an irreducible Hodge structure (of weight two), the assumption $f^{*}=$ id on $H^{2,0}(X)$ implies by Schur's lemma that $f^{*}=\mathrm{id}$ on $T(X)$. Together with $f^{*}=\mathrm{id}$ on $\operatorname{Pic}(X)$ this proves $f^{*}=$ id on the full cohomology $H^{*}(X, \mathbb{Z})$. By the global Torelli theorem, the latter is equivalent to $f=\mathrm{id}$. Eventually this shows that $\Phi_{G}^{\mathrm{CH}}([x])=f_{*}[x]=[x]$ for generic and hence all $x \in X$. Thus $\Phi_{G}^{\mathrm{CH}}=\mathrm{id}$ on $\mathrm{CH}^{2}(X)$.

To conclude we observe that $\Phi_{\mathcal{E}_{0}}\left(\mathcal{O}_{X}\right)$ deforms sideways to a spherical object in $\mathrm{D}^{\mathrm{b}}\left(\mathcal{X}_{K}\right)$, for $\mathcal{O}_{X}$ and $\mathcal{E}_{0}$ do. On the other hand, up to shift $\mathcal{O}_{\mathcal{X}_{K}^{\prime}}$ is the only spherical object in $\mathrm{D}^{\mathrm{b}}\left(\mathcal{X}_{K}^{\prime}\right)$ (cf. [12, Prop. 2.14]). Hence, up to shift, $\mathcal{O}_{X}$ is the only spherical object on $X$ that deforms sideways in the family $\mathcal{X}^{\prime}$ to a spherical object in $\mathrm{D}^{\mathrm{b}}\left(\mathcal{X}_{K}^{\prime}\right)$. Hence $\Phi_{\mathcal{E}_{0}}\left(\mathcal{O}_{X}\right) \simeq \mathcal{O}_{X}$ (up to shift), which in particular shows that $\Phi_{\mathcal{E}_{0}}^{\mathrm{CH}}\left(1,0, c_{X}\right)=\left(1,0, c_{X}\right)$.

Thus we have shown that $\Phi_{\mathcal{E}_{0}}^{\mathrm{CH}}$ acts as identity on $\mathrm{CH}^{0}(X) \oplus \mathrm{CH}^{2}(X)$. Moreover, it acts as $\left(\begin{array}{cc}\text { id } & 0 \\ * & \text { id }\end{array}\right)$ on $\mathrm{CH}^{1}(X) \oplus \mathrm{CH}^{2}(X)$, which will later be shown to be diagonal.

The proposition can also be used to derive information about the Mukai vectors in $\mathrm{CH}^{*}(X)$ of spherical objects having the same Mukai vector in cohomology. This is the following 
Corollary 2.6. If $E, E^{\prime} \in \mathrm{D}^{\mathrm{b}}(X)$ are two spherical objects with $v(E)=v\left(E^{\prime}\right) \in$ $\widetilde{H}(X, \mathbb{Z})$, then

$$
v^{\mathrm{CH}}(E)=v^{\mathrm{CH}}\left(E^{\prime}\right) \in \mathrm{CH}^{*}(X) .
$$

Proof. Write $v(E)=(r, \ell, s)=v\left(E^{\prime}\right)$. Let us first reduce to the case that $r \neq 0$. Suppose $r=0$, then $\ell \neq 0$. Then let $\Phi=T_{\mathcal{O}_{X}} \circ(L \otimes())$ and use $\Phi \circ T_{E} \simeq T_{\Phi(E)} \circ \Phi$ (see e.g. [10, Lemma 8.21]), which holds for any Fourier-Mukai equivalence $\Phi$ and any spherical object $E$. Thus $T_{\Phi(E)}^{H}=T_{\Phi\left(E^{\prime}\right)}^{H}$ and the assertion $v^{\mathrm{CH}}(E)=v^{\mathrm{CH}}\left(E^{\prime}\right)$ is clearly equivalent to $v^{\mathrm{CH}}(\Phi(E))=v^{\mathrm{CH}}\left(\Phi\left(E^{\prime}\right)\right)$. If $L$ is chosen such that $\left(\mathrm{c}_{1}(L) . \ell\right) \ll 0$, then the spherical object $\Phi(E)$ has positive rank.

Now apply Proposition 2.5 to the class $c_{X}$ to deduce $T_{E}^{\mathrm{CH}}\left(c_{X}\right)=T_{E^{\prime}}^{\mathrm{CH}}\left(c_{X}\right)$. Both sides can be explicitly computed, which yields $c_{X}-r v^{\mathrm{CH}}(E)=c_{X}-r v^{\mathrm{CH}}\left(E^{\prime}\right)$ and hence $v^{\mathrm{CH}}(E)=v^{\mathrm{CH}}\left(E^{\prime}\right)$.

Proof of Theorem 2.3 Suppose again that $\Phi_{\mathcal{E}}$ is an autoequivalence of $\mathrm{D}^{\mathrm{b}}(X)$ with $\Phi_{\mathcal{E}}^{H}=$ id. By Proposition 2.5 we know already that $\Phi_{\mathcal{E}}^{\mathrm{CH}}$ is the identity on $\mathrm{CH}^{0}(X) \oplus \mathrm{CH}^{2}(X)$. Thus it remains to show that $\Phi_{\mathcal{E}}^{\mathrm{CH}}\left(\mathrm{c}_{1}(L)\right)$ of an arbitrary line bundle $L$ has no component in $A(X)$. The image $L^{\prime}:=\Phi_{\mathcal{E}}(L)$ of a line bundle $L$ is a spherical object and since $\Phi_{\mathcal{E}}^{H}=\mathrm{id}$, one has $v(L)=v\left(L^{\prime}\right)$. By Corollary 2.6 this implies $v^{\mathrm{CH}}(L)=v^{\mathrm{CH}}\left(L^{\prime}\right)$ and hence $\Phi_{\mathcal{E}}^{\mathrm{CH}}\left(\mathrm{c}_{1}(L)\right) \in \mathrm{CH}^{1}(X)$.

As done already in the proof above, Theorem 2.3 can be reformulated in terms of autoequivalences. Since the kernel of the cohomology representation of $\operatorname{Aut}\left(\mathrm{D}^{\mathrm{b}}(X)\right)$ is essentially the only remaining mystery in this context, we state this explicitly as

Corollary 2.7. Let X be a smooth complex projective K3 surface and denote by

$$
\rho^{\mathrm{CH}}: \operatorname{Aut}\left(\mathrm{D}^{\mathrm{b}}(X)\right) \rightarrow \operatorname{Aut}\left(\mathrm{CH}^{*}(X)\right) \quad \text { and } \rho^{H}: \operatorname{Aut}\left(\mathrm{D}^{\mathrm{b}}(X)\right) \rightarrow \operatorname{Aut}(\tilde{H}(X, \mathbb{Z}))
$$

the natural representation $\Phi \mapsto \Phi^{\mathrm{CH}}$ resp. $\Phi \mapsto \Phi^{H}$. Then $\operatorname{ker}\left(\rho^{\mathrm{CH}}\right)=\operatorname{ker}\left(\rho^{H}\right)$.

Proof. The inclusion $\operatorname{ker}\left(\rho^{\mathrm{CH}}\right) \subset \operatorname{ker}\left(\rho^{H}\right)$ is obvious and the other one follows from the proposition.

Remark 2.8. In [7] Bridgeland suggests the following explicit description of this kernel. He conjectures $\operatorname{ker}\left(\rho^{H}\right)=\pi_{1}\left(\mathcal{P}_{0}^{+}(X)\right)$, for a certain period domain $\mathcal{P}_{0}^{+}(X)$ defined in terms of the algebraic part of $\widetilde{H}(X, \mathbb{Z})$. In particular, the conjecture says that $\operatorname{ker}\left(\rho^{H}\right)$ is spanned by the square $T^{2}=[2]$ of the shift functor and the squares $T_{E}^{2}$ of all spherical twists $T_{E}$. (In fact, spherical twists associated to spherical sheaves should suffice.)

As explained above, the conjectural generators $T^{2}$ and $T_{E}^{2}$ of $\operatorname{ker}\left(\rho^{H}\right)$ act trivially on $\mathrm{CH}^{*}(X)$. In this sense, the corollary provides non-trivial evidence for Bridgeland's conjecture.

Remark 2.9. The corollary appears interesting also in the light of another open conjecture due to Bloch (see [3]), which for the case of surfaces reads as follows: Consider a surface $X$ and a cycle $\Gamma \in \mathrm{CH}^{2}(X \times X)$ with its induced natural endomorphisms $[\Gamma]_{*}^{2,0}$ of 
$H^{0}\left(X, \Omega_{X}^{2}\right)$ and $[\Gamma]_{*}$ of $\mathrm{CH}^{2}(X)$. In general the latter does not respect the natural filtration $\operatorname{ker}\left(\mathrm{alb}_{X}\right) \subset A(X) \subset \mathrm{CH}^{2}(X)$, but induces an endomorphism $\operatorname{gr}[\Gamma]_{*}$ of the graded object $\operatorname{ker}\left(\operatorname{alb}_{X}\right) \oplus \operatorname{Alb}(X) \oplus \mathbb{Z}$. Then Bloch conjectures that $[\Gamma]_{*}^{2,0}=0$ if and only if $\operatorname{gr}[\Gamma]_{*}$ is trivial on $\operatorname{ker}\left(\mathrm{alb}_{X}\right)$ (see also [24, Ch. 11]). Note that for a K3 surface $X$ the graded object is just $A(X) \oplus \mathbb{Z}$.

If $\Phi_{\mathcal{E}}: \mathrm{D}^{\mathrm{b}}(X) \stackrel{\sim}{\rightarrow} \mathrm{D}^{\mathrm{b}}(X)$ is a Fourier-Mukai autoequivalence of a K3 surface $X$ such that $\Phi_{\mathcal{E}}^{H}=$ id, then $\Gamma:=v^{\mathrm{CH}}(\mathcal{E})-[\Delta]$ acts trivially on cohomology and, in particular, on $H^{0}\left(X, \Omega_{X}^{2}\right)$. Bloch's conjecture would thus say that $\operatorname{gr}[\Gamma]_{*}$ is trivial on $A(X)=\operatorname{ker}\left(\mathrm{alb}_{X}\right)$. And indeed, by Corollary 2.7 this holds true, as we in fact have $\Gamma=0$

Note that Bloch's conjecture would actually say that $\Phi_{\mathcal{E}}^{H}=$ id on $H^{0}\left(X, \Omega_{X}^{2}\right)$ is sufficient to conclude $\Phi_{\mathcal{E}}^{\mathrm{CH}}=$ id on $A(X)$, but our techniques fail to prove this. In fact, it seems even unknown whether any symplectomorphism $f \in \operatorname{Aut}(X)$ induces the identity on $A(X)$, i.e. whether for a symplectomorphism $f$ any point $x \in X$ is rationally equivalent to its image $f(x)$ (cf. comments in the proof of Proposition 2.5].

\section{Mukai vectors of spherical objects}

The goal of this section is to prove that for any spherical object $E \in \mathrm{D}^{\mathrm{b}}(X)$ on a smooth complex projective $\mathrm{K} 3$ surface $X$ with Picard number $\rho(X) \geq 2$ the Mukai vector $v^{\mathrm{CH}}(E) \in \mathrm{CH}^{*}(X)$ is contained in the Beauville-Voisin subring $R(X) \subset \mathrm{CH}^{*}(X)$. The main results (Corollaries 3.3 and 3.4) should also hold for K3 surfaces with $\rho(X)=1$, but we can only prove it under additional conditions on the numerical invariants of $E$.

Remark 3.1. As noted earlier, the original result in [5] can be seen as the special case that the spherical object $E$ is a line bundle $L \in \operatorname{Pic}(X)$. Indeed, if

$$
v^{\mathrm{CH}}\left(L^{k}\right)=\left(1, k \mathrm{c}_{1}(L), k^{2} \mathrm{c}_{1}(L)^{2} / 2+\mathrm{c}_{2}(X) / 24\right) \in R(X),
$$

then necessarily $\mathrm{c}_{1}(L)^{2} \in R(X)$ and $\mathrm{c}_{2}(X) \in R(X)$. However, it should be emphasized that our methods do not provide an alternative proof of the results in [5].

In this section we shall explain how to reduce the proof of Theorem 1 to a generalization of the Beauville-Voisin result from line bundles to higher rank spherical vector bundles. The proof of the following crucial result is postponed to the next section.

Proposition 3.2. Let $X$ be a smooth complex projective $K 3$ surface and let $E$ be a spherical vector bundle on $X$. Suppose that one of the following conditions hold:

(i) $\rho(X) \geq 2$ or

(ii) $\operatorname{Pic}(X)=\mathbb{Z} H$ and $v(E)=(r, k H, s)$ with $k \equiv \pm 1(r)$.

Then $v^{\mathrm{CH}}(E) \in R(X)$. 
This proposition is expected to hold without any restriction on $X$ or the spherical vector bundle $E$. However, it does not generalize to $\mu$-stable vector bundles, i.e. the Mukai vector of a general non-rigid $\mu$-stable vector bundle $E$ is certainly not contained in $R(X)$.

The following consequence of Proposition 3.2 proves Theorem 1.

Corollary 3.3. Let $E \in \mathrm{D}^{\mathrm{b}}(X)$ be a spherical object on a smooth projective $K 3$ surface $X$. Suppose that either

(i) $\rho(X) \geq 2$ or

(ii) $\operatorname{Pic}(X)=\mathbb{Z} H$ with $v(E)=(r, k H, s)$ with $k \equiv \pm 1(r)$.

Then $v^{\mathrm{CH}}(E) \in R(X)$.

Proof. Let $E \in \mathrm{D}^{\mathrm{b}}(X)$ be spherical. Write $v(E)=(r, \ell, s)$. Clearly, $v^{\mathrm{CH}}(E) \in R(X)$ is equivalent to $v^{\mathrm{CH}}(E[1])=-v^{\mathrm{CH}}(E) \in R(X)$. Hence we may assume $r \geq 0$.

If $r>0$, then as proved in [14] there exists a spherical locally free sheaf $E^{\prime}$ on $X$ with $v\left(E^{\prime}\right)=v(E)$. (In fact any torsion free spherical sheaf is automatically locally free, as was already observed by Mukai in [19].) Now apply Proposition 3.2 to $E^{\prime}$ which yields $v^{\mathrm{CH}}\left(E^{\prime}\right) \in R(X)$. But by Corollary 2.6, we know that $v^{\mathrm{CH}}(E)=v^{\mathrm{CH}}\left(E^{\prime}\right)$ for any two numerically equivalent spherical objects $E, E^{\prime} \in \mathrm{D}^{\mathrm{b}}(X)$.

The case $r=0$ is straightforward. First, by applying $T_{\mathcal{O}_{X}}$ we reduce to the case that also $s=0$ and hence $v(E)=(0, \ell, 0)$ with $\ell$ a $(-2)$-class, which we may assume to be effective. Thus $T_{E}^{H}=s_{\ell}$, the reflection in $\ell^{\perp}$. The Weyl group $W_{X}$ generated by reflections $s_{\delta}$ for all effective $(-2)$-classes $\delta$ is known to be generated by reflections associated to nodal classes, i.e. when $\delta$ is represented by a smooth rational curve. Thus $s_{\ell}$ can be written as a composition of finitely many reflections $s_{\left[C_{i}\right]}$, where the curves $C_{i} \subset X$ are smooth and rational. Then use that $v^{\mathrm{CH}}(E) \in R(X)$ is equivalent to $T_{E}^{\mathrm{CH}}(R(X))$ $=R(X)$, because $T_{E}^{\mathrm{CH}}$ is the reflection in the hyperplane $v^{\mathrm{CH}}(E)^{\perp}$, and that $T_{E}^{H}=s_{\ell}=$ $\prod s_{\left[C_{i}\right]}$ implies $T_{E}^{\mathrm{CH}}=\prod T_{\mathcal{O}_{C_{i}}(-1)}^{\mathrm{CH}}\left(\right.$ Theorem 2.3p. Clearly, $v^{\mathrm{CH}}\left(\mathcal{O}_{C_{i}}(-1)\right) \in R(X)$ and hence $T_{\mathcal{O}_{C_{i}}(-1)}^{\mathrm{CH}}(R(X))=R(X)$.

Let us now show that Theorem 1 implies Theorem 2, which we state again as

Corollary 3.4. Suppose $X$ and $X^{\prime}$ are smooth complex projective K3 surfaces with Picard number $\rho(X) \geq 2$. If $\Phi_{\mathcal{E}}: \mathrm{D}^{\mathrm{b}}(X) \stackrel{\sim}{\rightarrow} \mathrm{D}^{\mathrm{b}}\left(X^{\prime}\right)$ is a Fourier-Mukai equivalence, then the induced map $\Phi_{\mathcal{E}}^{\overline{\mathrm{CH}}}: \mathrm{CH}^{*}(X) \stackrel{\sim}{\rightarrow} \mathrm{CH}^{*}\left(X^{\prime}\right)$ respects the Beauville-Voisin subring, i.e.

$$
\Phi_{\mathcal{E}}^{\mathrm{CH}}(R(X))=R\left(X^{\prime}\right) .
$$

Proof. The Mukai vectors $v^{\mathrm{CH}}(L) \in \mathrm{CH}^{*}(X)$ of all line bundles $L \in \operatorname{Pic}(X) \operatorname{span} R(X)$. The images $E:=\Phi_{\mathcal{E}}(L) \in \mathrm{D}^{\mathrm{b}}\left(X^{\prime}\right)$ are not necessarily (shifted) line bundle again, but they are spherical objects in $\mathrm{D}^{\mathrm{b}}\left(X^{\prime}\right)$. Since $\rho(X) \geq 2$ and hence $\rho\left(X^{\prime}\right) \geq 2$, Corollary 3.3 i) applies. Thus $\Phi_{\mathcal{E}}^{\mathrm{CH}}\left(v^{\mathrm{CH}}(L)\right)=v^{\mathrm{CH}}(E) \in R\left(X^{\prime}\right)$.

Remark 3.5. The most interesting special case is the one when $X^{\prime}$ is a fine moduli space of $\mu$-stable vector bundles and $\mathcal{E}$ is the universal bundle $\mathbb{E}$. In examples where both the 
moduli space $X^{\prime}$ and the universal bundle $\mathbb{E}$ are constructed explicitly, one can sometimes prove Corollary 3.4 directly (see e.g. [11] for explicit examples). If there was an argument proving the result for arbitrary universal bundles without first proving Corollary 3.3 , then the techniques of Section 2 would prove Corollary 3.4 more directly (and also in the case $\rho(X)=1)$.

To be more precise, let $E$ be any spherical object with $v(E)=(r, \ell, s)$ and $r>0$. Then $T_{E}^{H}(0,0,1)=-\left(r^{2}, r \ell, r s-1\right)$. Thus, if $T_{E}$ is composed with $\Phi_{\mathbb{E}[1]}$ where $\mathbb{E} \in \mathrm{D}^{\mathrm{b}}\left(X \times X^{\prime}\right)$ is the universal family of stable vector bundles with Mukai vector $\left(r^{2}, r \ell, r s-1\right)$, then $\left(\Phi_{\mathbb{E}[1]} \circ T_{E}\right)^{H}(0,0,1)=(0,0,1)$. By composing with a certain equivalence $\Psi$ that is a combination of spherical twists $T_{\mathcal{O}_{C}}$ (with $\mathbb{P}^{1} \simeq C \subset X$ ) and tensor products with line bundles, the Hodge isometry $\left(\Psi \circ \Phi_{\mathbb{E}[1]} \circ T_{E}\right)^{H}$ becomes graded. Moreover, it will respect the Kähler cone up to sign (see e.g. [10, Ch. 9] for details). Then by the global Torelli theorem $\left(\Psi \circ \Phi_{\mathbb{E}[1]} \circ T_{E}\right)^{H}= \pm f_{*}$ for some isomorphism $f$. Now use that $f_{*}^{\mathrm{CH}}$ and $\Psi^{\mathrm{CH}}$ preserve the Beauville-Voisin ring. Hence $T_{E}^{\mathrm{CH}}(R(X))=R(X)$ if and only if $\Phi_{\mathbb{E}}^{\mathrm{CH}}(R(X))=R\left(X^{\prime}\right)$, where $\mathbb{E}$ is a universal family of stable bundles of rank $r^{2}$.

\section{Spherical vector bundles: Proof of Proposition 3.2}

Let $C$ be a smooth irreducible complex projective curve of genus $g$. Recall that the BrillNoether locus $W_{d}^{r_{0}}(C) \subset \operatorname{Pic}^{d}(C)$ is the determinantal subvariety of all line bundles $A$ of degree $d$ with $h^{0}(C, A) \geq r_{0}+1$. The Brill-Noether number for these numerical invariants is by definition

$$
\rho\left(r_{0}, d, g\right):=g-\left(r_{0}+1\right)\left(g-d+r_{0}\right) .
$$

Classically (see [1]) one knows that $W_{d}^{r_{0}}(C)$ is non-empty whenever $\rho\left(r_{0}, d, g\right) \geq 0$. (Due to a result of Fulton and Lazarsfeld, it is also connected when $\rho\left(r_{0}, d, g\right)>0$, but this will not be used.) Moreover, for a generic curve $C$ the Brill-Noether number $\rho\left(r_{0}, d, g\right)$ is in fact the dimension of $W_{d}^{r_{0}}(C)$ when $\rho\left(r_{0}, d, g\right) \geq 0$ and $W_{d}^{r_{0}}(C)=\emptyset$ otherwise.

Central for our discussion is a result of Lazarsfeld [15] that shows that a generic smooth curve $C$ in an indecomposable linear system on a $\mathrm{K} 3$ surface is Brill-Noether general, i.e. the $W_{d}^{r_{0}}(C)$ have the expected dimension. Let us make precise which parts of [15] are really used.

Suppose $A \in W_{d}^{r_{0}}(C)$ satisfies

$h^{0}(C, A)=r_{0}+1$ and the line bundles $A$ and $A^{*} \otimes \omega_{C}$ are globally generated. (4.1)

If $C$ is embedded into a $\mathrm{K} 3$ surface $X$, one associates to $A$ the Lazarsfeld bundle $F_{C, A}$, which by definition is the kernel of the evaluation map $H^{0}(C, A) \otimes \mathcal{O}_{X} \rightarrow A$. Here $A$ is viewed as a sheaf on $X$ supported on $C$. Thus, there is a short exact sequence

$$
0 \rightarrow F_{C, A} \rightarrow H^{0}(C, A) \otimes \mathcal{O}_{X} \rightarrow A \rightarrow 0
$$


and it is not difficult to see that $F_{C, A}$ is really locally free. Dualizing yields an exact sequence

$$
0 \rightarrow H^{0}(C, A)^{*} \otimes \mathcal{O}_{X} \rightarrow F_{C, A}^{*} \rightarrow A^{*} \otimes \omega_{C} \rightarrow 0 .
$$

The crucial result for our discussion is the following observation.

Lemma 4.1 ([15, Lemma 1.3]). If $|C|$ is indecomposable, i.e. $|C|$ does not contain any reducible curves, then the bundle $F_{C, A}$ is simple.

Clearly, the assumption on $|C|$ is satisfied if $\operatorname{Pic}(X)$ is generated by $\mathcal{O}(C)$ and we shall restrict to this case. So from now on let $X$ be a complex projective K3 surface with $\rho(X)=1$, let $H \in \operatorname{Pic}(X)$ be the ample generator and write $(H . H)=2 g-2$. Then the generic curve $C \in|H|$ is smooth of genus $g$. (Indeed, by Bertini it suffices to show that $|H|$ has no base points and according to [22, Cor. 3.2] there are no base points outside the fixed components which do not exist, because $|H|$ is indecomposable.)

Now choose $r_{0}$ and $d$ such that

$$
d<g+r_{0} \quad \text { and } \quad \rho\left(r_{0}, d, g\right)=0 .
$$

We will only need the following immediate consequence of [15]:

Proposition 4.2. For generic $C \in|H|$ there exists a line bundle $A \in W_{d}^{r_{0}}(C)$ satisfying 4.1 .

Proof. In fact we will show that for a generic curve $C \in|H|$ any $A \in W_{d}^{r_{0}}(C)$ satisfies 4.1 . Since $\rho\left(r_{0}, d, g\right)=0$ and hence $W_{d}^{r_{0}}(C) \neq \emptyset$ (for any smooth $C$ ), this proves the assertion.

Let $C \in|H|$ be generic and let $A \in W_{d}^{r_{0}}(C)$. We first check $h^{0}(C, A)=r_{0}+1$. If not, then $W_{d}^{r_{0}+1}(C) \neq \emptyset$. On the other hand, by our assumption 4.3 we have $\rho\left(r_{0}+1, d, g\right)=$ $\rho\left(r_{0}, d, g\right)-\left(g-d+r_{0}+1\right)-\left(r_{0}+2\right)=0+d-g-2 r_{0}-3<0$ and thus $W_{d}^{r_{0}+1}(C)=\emptyset$, as the generic smooth curve in $|H|$ is Brill-Noether general according to [15].

Next, for generic $C \in|H|$ any $A \in W_{d}^{r_{0}}(C)$ is globally generated. Indeed, otherwise $W_{d-1}^{r_{0}}(C) \neq \emptyset$. This would again contradict that $C$ is Brill-Noether general, for $\rho\left(r_{0}, d-1, g\right)=\rho\left(r_{0}, d, g\right)-\left(r_{0}+1\right)<0$.

The calculation for $A^{*} \otimes \omega_{C}$ is similar. First observe $A^{*} \otimes \omega_{C} \in W_{2 g-2-d}^{r_{0}-d-1+g}(C)$ and $h^{0}\left(C, A^{*} \otimes \omega_{C}\right)=r_{0}-d+g$ by Serre duality and Riemann-Roch. Then, using the assumption $d<g+r_{0}$ in (4.3) (so far $d<g+2 r_{0}$ was enough), one checks $\rho\left(r_{0}-d-1+g, 2 g-2-d-1, g\right)<0$ and hence $W_{2 g-2-d-1}^{r_{0}-d-1+g}(C)=\emptyset$. The latter shows in particular that $A^{*} \otimes \omega_{C}$ is globally generated.

We continue to assume $\operatorname{Pic}(X)=\mathbb{Z} H$. Consider a spherical bundle $E$ on $X$ and let $v(E)=(r, k H, s)$ with $k \equiv \pm 1(r)$. By tensoring with powers of $H$ and dualizing we can modify $E$ such that $k=1$. As these operations do not affect whether $v^{\mathrm{CH}}(E) \in R(X)$, we will assume henceforth that $k=1$. Since $E$ is spherical, one has $(H . H)-2 r s=-2$ or, in other words, $r s=g$. 
Next we would like to relate $E$ to a particular Lazarsfeld bundle, but a priori it is not clear that $E$ fits in a short exact sequence of the form $0 \rightarrow \mathcal{O}_{X}^{r} \rightarrow E \rightarrow M \rightarrow 0$ with $M$ a line bundle on a generic $C \in|H|$ (cf. (4.2)). However, we will see that this is possible for the right choice of $r_{0}$ and $d$. To be more precise, let

$$
d:=g-1-s+r \quad \text { and } \quad r_{0}:=r-1 .
$$

(If the wished-for exact sequence $0 \rightarrow \mathcal{O}_{X}^{r} \rightarrow E \rightarrow M \rightarrow 0$ is of the form 4.2, then the Riemann-Roch formula $\chi(M)=-d+g-1$ together with $\chi(M)=\chi(E)-2 r=s-r$ dictates this choice.)

A straightforward computation reveals that with this choice $\rho\left(r_{0}, d, g\right)=g-r s=0$ and $d<g+r_{0}$. The latter is equivalent to $s>0$, which follows from $g=r s$ and $r>0$. Thus Proposition 4.2 applies and we find for generic $C \in|H|$ a line bundle $A \in W_{d}^{r_{0}}(C)$ satisfying 4.3 . This then yields a short exact sequence of the form 4.2). Moreover, $F_{C, A}^{*}$ is simple by Lemma 4.1 and $v\left(F_{C, A}^{*}\right)=(r, H, s)=v(E)$.

By [19. Prop. 3.14], any spherical bundle on a K3 surface with Picard number one is $\mu$-stable. Mukai also proves that rigid $\mu$-stable vector bundles with given Mukai vector are unique (see also [11, Thm. 6.16]). Hence $E \simeq F_{C, A}^{*}$.

Thus as a consequence of [15] we proved

Corollary 4.3. Let $E$ be a spherical bundle on a $K 3$ surface $X$ with $\operatorname{Pic}(X)=\mathbb{Z} H$ and such that $v(E)=(r, H, s)$. Then for any generic smooth curve $C \in|H|$ there exists a line bundle $M$ on $C$ and a short exact sequence

$$
0 \rightarrow \mathcal{O}_{X}^{r} \rightarrow E \rightarrow M \rightarrow 0 .
$$

Remark 4.4. Corollary 2.6 which also works for $\rho(X)>1$, shows that in any case $v^{\mathrm{CH}}(E)=v^{\mathrm{CH}}\left(F_{C, A}^{*}\right)$. So we do not actually need $E \simeq F_{C, A}^{*}$, but only that the simple $F_{C, A}^{*}$ exists.

The rough idea of the next step is to let the smooth generic curve $C$ degenerate to a rational curve $C_{0} \in|H|$, which always exists due to Mumford (cf. [18] or [2]). At the same time, $M$ will deform to a sheaf $M_{0}$ supported on $C_{0}$. Since the right hand side in $v^{\mathrm{CH}}(M)=v^{\mathrm{CH}}(E)-v^{\mathrm{CH}}\left(\mathcal{O}_{X}^{r}\right)$ stays constant in the process, one also has $v^{\mathrm{CH}}\left(M_{0}\right)=$ $v^{\mathrm{CH}}(E)-v^{\mathrm{CH}}\left(\mathcal{O}_{X}^{r}\right)$. But now $M_{0}$ is supported on the rational curve $C_{0} \subset X$ and by [5] this implies $v^{\mathrm{CH}}\left(M_{0}\right) \in R(X)$. Hence $v^{\mathrm{CH}}(E) \in R(X)$.

This can be made rigorous as follows: Consider $Z:=\operatorname{Gr}\left(r, H^{0}(X, E)\right)$ and the nonempty Zariski open subset $U \subset Z$ of all subspaces $V \subset H^{0}(X, E)$ such that $V \otimes \mathcal{O}_{X} \rightarrow$ $E$ is injective with cokernel $M$ being a line bundle on a smooth curve $C$. Then $C \in|H|$, for $\mathrm{c}_{1}(E)=H$. By Corollary 4.3 the set $U$ is not empty and in fact the composition

$$
U \rightarrow \operatorname{Pic}^{d}(\mathcal{C} /|H|) \rightarrow|H|
$$

is dominant. Here $\mathcal{C} \rightarrow|H|$ is the linear system together with its universal curve and $\operatorname{Pic}^{d}(\mathcal{C} /|H|) \rightarrow|H|$ denotes the compactified relative Jacobian variety (or Simpson's moduli space of stable pure sheaves). 
The morphism $U \rightarrow \operatorname{Pic}^{d}(\mathcal{C} /|H|)$ can be compactified to a morphism $\varphi: Z^{\prime} \rightarrow$ $\operatorname{Pic}^{d}(\mathcal{C} /|H|)$ where $Z^{\prime}$ is some projective variety containing $U$ as a dense open subset. For the following, we can assume that the universal sheaf $\mathcal{M}$ on $\operatorname{Pic}^{d}(\mathcal{C} /|H|) \times X$ exists, otherwise pass to some projective variety dominating $\operatorname{Pic}^{d}(\mathcal{C} /|H|)$. Then the pull-back $\mathcal{N}:=(\varphi \times \mathrm{id})^{*} \mathcal{M}$ on $Z^{\prime} \times X$ has the property that $v^{\mathrm{CH}}\left(\mathcal{N}_{t}\right)=v^{\mathrm{CH}}(E)-v^{\mathrm{CH}}\left(\mathcal{O}_{X}^{r}\right)$ for any closed point $t \in U$. Hence also $v^{\mathrm{CH}}\left(\mathcal{N}_{t_{0}}\right)=v^{\mathrm{CH}}(E)-v^{\mathrm{CH}}\left(\mathcal{O}_{X}^{r}\right)$ for any closed point $t_{0} \in Z^{\prime}$ in the boundary.

Remark 4.5. This last argument makes use of the specialization map for Chow groups. Consider first a family $\mathcal{X} \rightarrow S$ over a smooth irreducible curve. Let $t \in S$ be a closed point and $\eta \in S$ be the generic point. Denote by $\mathcal{X}_{t}$ and $\mathcal{X}_{\eta}$ the corresponding fibres, which we regard as varieties over $k(t)$ resp. $k(\eta)=K(S)$. The closure of any cycle on $\mathcal{X}_{\eta}$ yields a cycle on $\mathcal{X}$ which can then be restricted to the closed fibre $\mathcal{X}_{t}$. Rational equivalence is preserved in the process, so that we get the specialization map

$$
\mathrm{CH}^{*}\left(\mathcal{X}_{\eta}\right) \rightarrow \mathrm{CH}^{*}\left(\mathcal{X}_{t}\right)
$$

See [8, Ch. 20] for details when $S$ is the spectrum of a discrete valuation ring with the two points $t$ and $\eta$. For an arbitrary (smooth and irreducible) base $S$ one constructs by recurring blow-ups (see e.g. [9, II, Exer. 4.12]) a morphism $\operatorname{Spec}(R) \rightarrow S$, with $R$ a discrete valuation ring, mapping the closed (resp. generic) point to $t$ (resp. $\eta$ ). Then pull back the family $\mathcal{X}$ to $\operatorname{Spec}(R)$ and apply the construction for discrete valuation rings. Note that by construction for any cycle $\alpha \in \mathrm{CH}^{*}(\mathcal{X})$ the restriction $\alpha_{t} \in \mathrm{CH}^{*}\left(\mathcal{X}_{t}\right)$ equals the image under the specialization map of the restriction $\alpha_{\eta}$ (cf. [8, 20.3.1]).

This specialization technique applies to our case, as the short exact sequences associated to any $V \subset H^{0}(X, E)$ in $U$ glue to a short exact sequence over $U$ and hence over the generic point $\eta \in U \subset Z^{\prime}$. Thus one has $v^{\mathrm{CH}}\left(\mathcal{N}_{\eta}\right)=v^{\mathrm{CH}}\left(E_{\eta}\right)-v^{\mathrm{CH}}\left(\mathcal{O}_{X_{\eta}}^{r}\right)$, where $E_{\eta}$ is obtained by base change $X_{\eta}:=X \times \mathbb{C} \eta \rightarrow X$.

Since $U \rightarrow|H|$ is dominant, there exists a closed point $t_{0} \in Z^{\prime}$ which under

$$
Z^{\prime} \rightarrow \operatorname{Pic}^{d}(\mathcal{C} /|H|) \rightarrow|H|
$$

maps to a closed point corresponding to an irreducible rational curve $C_{0} \in|H|$. Thus $\mathcal{N}_{t_{0}}$ is supported on an irreducible rational curve. But then $v^{\mathrm{CH}}\left(\mathcal{N}_{t_{0}}\right) \in R(X)$ and hence $v^{\mathrm{CH}}(E) \in R(X)$. Thus we have proved

Proposition 4.6. Let $X$ be a $K 3$ surface with $\operatorname{Pic}(X)=\mathbb{Z} H$. If $E$ is a spherical bundle with $v(E)=(r, k H, s)$ and $k \equiv \pm 1(r)$, then $v^{\mathrm{CH}}(E) \in R(X)$.

Remark 4.7. Note that the above arguments also work for $\rho(X) \geq 2$ whenever $U \rightarrow|H|$ is dominant, but without using Lazarsfeld's result this seems difficult.

Remark 4.8. There is an alternative argument going back to Mumford that would replace the degeneration argument. One can show that the set of effective cycles $Z \in S^{n}(X)$ rationally equivalent to a given one $Z_{0} \in \mathrm{S}^{n}(X)$ forms a countable union of irreducible 
Zariski closed subsets. The countability stems from the fact that the number of irreducible components of the Hilbert scheme of all subvarieties is countable. See [24, Ch. 22] for an account.

By taking sections of the line bundles $\mathcal{N}_{t}$ one obtains effective cycles on $X$, which for all $t$ in the open subset $U$ are rationally equivalent to each other (and to $c_{2}(E)$ ). But then this holds for any cycle in the closure of the image of $U \rightarrow \mathrm{S}^{n}(X)$, which necessarily contains a cycle that is contained in a rational curve.

In order to fully prove Proposition 3.2 , it remains to treat the case $\rho(X) \geq 2$. There are essentially two arguments involved:

(i) Show that on a K3 surface $X$ with $\rho(X) \geq 2$ tensoring with line bundles and dualizing brings the Mukai vector of any spherical bundle $E$ into the form $v(E)=(r, H, s)$, where $H$ is a primitive ample line bundle.

(ii) View any polarized $\mathrm{K} 3$ surface $(X, H)$ with $\rho(X) \geq 2$ as a degeneration of a polarized K3 surface of Picard number one. Then use Proposition 4.6 and a degeneration argument.

The first question is purely numerical: Suppose $E$ is a spherical vector bundle with $v(E)=\left(r, \mathrm{c}_{1}(L), s\right)$. Write $\mathrm{c}_{1}(L)=k \ell$ for some primitive $\ell \in \mathrm{NS}(X)$ and $k \in \mathbb{Z}$. As $E$ is spherical, one has $k^{2}(\ell . \ell)-2 r s=-2$. Thus $k$ and $r$ are coprime, for $(\ell . \ell)$ is even. Assuming $\rho(X) \geq 2$, there exists a line bundle $M \in \operatorname{Pic}(X)$ such that $\mathrm{c}_{1}(E \otimes M)=$ $k \ell+r c_{1}(M)$ is primitive and ample. (Indeed, complete $e_{1}:=\ell$ to a basis $e_{1}, \ldots, e_{\rho}$ of $\mathrm{NS}(X)$ and choose $M$ such that $\mathrm{c}_{1}(M)=\sum a_{i} e_{i}$ with $a_{2}= \pm r^{n}$. Then $k+r a_{1}$ and $r a_{2}= \pm r^{n+1}$ are coprime and for $n \gg 0$ the coefficients $a_{1}, a_{3}, \ldots, a_{\rho}$ can be chosen such that $k e_{1}+r c_{1}(M)$ is contained in the ample cone, which is open.)

Since $v^{\mathrm{CH}}(E) \in R(X)$ is equivalent to $v^{\mathrm{CH}}(E \otimes M) \in R(X)$, it suffices to consider the following situation: $(X, H)$ is a polarized $\mathrm{K} 3$ surface with $H$ primitive and $E$ is a spherical vector bundle on $X$ with $\operatorname{det}(E)=H$.

In step (ii) we choose a smooth projective family of polarized $\mathrm{K} 3$ surfaces $\pi:(\mathcal{X}, \mathcal{H})$ $\rightarrow D$ over a curve $D$, such that a distinguished fibre, say over the closed point $0 \in D$, is $(X, H)$, i.e. $\mathcal{X}_{0} \simeq X$ and $\mathcal{H}_{0}:=\left.\mathcal{H}\right|_{\mathcal{X}_{0}} \simeq H$, and such that the general fibre has Picard number one. More precisely, for all except countably many closed points $t \in D$ one has $\rho\left(\mathcal{X}_{t}\right)=\mathbb{Z} \mathcal{H}_{t}$.

The obstructions to deform the spherical bundle $E$ on the central fibre $X$ to a bundle on the nearby fibres in $\mathcal{X} \rightarrow D$ are contained in $\operatorname{Ext}_{X}^{2}(E, E)$ and their traces are the obstructions to deform $\operatorname{det}(E)=H$ sideways. Since $\mathcal{H}$ exists, the latter must be trivial. As $E$ is spherical, the trace free part of $\operatorname{Ext}_{X}^{2}(E, E)$ is trivial and hence $E$ deforms to a vector bundle $\mathcal{E}$ on $\mathcal{X}$, possibly after shrinking $D$. So $\mathcal{E}_{0}:=\left.\mathcal{E}\right|_{\mathcal{X}_{0}} \simeq E$ and, by semicontinuity (shrink $D$ again if necessary), the restriction $\mathcal{E}_{t}$ to any other fibre $\mathcal{X}_{t}$ is spherical as well. A degeneration argument then yields the following result, which completes the proof of Proposition 3.2

Proposition 4.9. Suppose $E$ is a spherical vector bundle on a $K 3$ surface with $\rho(X) \geq 2$. Then $v^{\mathrm{CH}}(E) \in R(X)$. 
Proof. Again, there are two ways of proving this (cf. Remark 4.5 and the discussion following it). One can argue as Mumford and say that either $v^{\mathrm{CH}}\left(\mathcal{E}_{t}\right) \in R\left(\mathcal{X}_{t}\right)$ for all closed points $t \in D$ or for only a countable number of them (cf. Remark 4.8 which one easily adapts to the relative setting). Since over $\mathbb{C}$ the number of closed points $t \in D$ with $\rho\left(\mathcal{X}_{t}\right)=1$ is uncountable and for them $v^{\mathrm{CH}}\left(\mathcal{E}_{t}\right) \in R\left(\mathcal{X}_{t}\right)$ by Proposition 4.6 , we must have $v^{\mathrm{CH}}\left(\mathcal{E}_{t}\right) \in R\left(\mathcal{X}_{t}\right)$ for all closed points $t \in D$ and in particular for $t=0$. Hence, $v^{\mathrm{CH}}(E) \in R(X)$.

An alternative argument would be the following. Consider the relative Grassmannian $\operatorname{Gr}\left(r, \pi_{*} \mathcal{E}\right)$ with fibres $\operatorname{Gr}\left(r, H^{0}\left(\mathcal{X}_{t}, \mathcal{E}_{t}\right)\right.$ ) (at least over a non-empty open subset of $D$ to which we tacitly restrict). Then let $\mathcal{U} \subset \operatorname{Gr}\left(r, \pi_{*} \mathcal{E}\right)$ be the open subset of subspaces $V \subset$ $H^{0}\left(\mathcal{X}_{t}, \mathcal{E}_{t}\right)$ inducing short exact sequences of the form $0 \rightarrow V \otimes \mathcal{O}_{\mathcal{X}_{t}} \rightarrow \mathcal{E}_{t} \rightarrow M \rightarrow 0$ with $M$ a line bundle on some smooth curve on $\mathcal{X}_{t}$ in the ample linear system $\left|\mathcal{H}_{t}\right|$. As explained earlier, if $\rho\left(\mathcal{X}_{t}\right)=1$, the natural morphism $\varphi_{t}: \mathcal{U}_{t} \rightarrow\left|\mathcal{H}_{t}\right|$ is dominant. But this is an open condition. Hence $\varphi_{t}$ is actually surjective on a Zariski open subset of closed points $t \in D$ and thus over the generic point $\eta \in D$. Then imitate the degeneration argument for the linear system on $\mathcal{X}_{\eta}$, which shows that $v^{\mathrm{CH}}\left(\mathcal{E}_{\eta}\right) \in R\left(\mathcal{X}_{\eta}\right)$. Now use the specialization map $\mathrm{CH}\left(\mathcal{X}_{\eta}\right) \rightarrow \mathrm{CH}\left(\mathcal{X}_{0}\right)$.

\section{K3 surfaces over number fields}

The situation changes dramatically if instead of $\mathrm{K} 3$ surfaces over $\mathbb{C}$ one considers smooth projective K3 surfaces defined over a number field or over $\overline{\mathbb{Q}}$. In fact, a general conjecture of Beilinson and Bloch (see [4, 21]) applied to this case can be stated as follows:

Conjecture 5.1. If $X$ is a smooth projective $K 3$ surface over a number field $K$ or $\overline{\mathbb{Q}}$, then

$$
\operatorname{deg}: \mathrm{CH}^{2}(X) \otimes \mathbb{Q} \stackrel{\sim}{\rightarrow} \mathbb{Q} .
$$

How does this compare to [5] and to the results of the previous sections? Choose an embedding $K \subset \mathbb{C}$ and let $X_{\mathbb{C}}:=X \times_{K} \mathbb{C}$ be the induced complex K3 surface. A folklore argument shows that for arbitrary $X$ the kernel of the natural map

$$
\mathrm{CH}^{*}(X) \rightarrow \mathrm{CH}^{*}\left(X_{\mathbb{C}}\right)
$$

is torsion. Thus, Conjecture 5.1 can be rephrased as

Conjecture 5.2 (Bloch-Beilinson for $\mathrm{K} 3$ surfaces). If $X$ is a smooth projective K3 surface over a number field $K \subset \mathbb{C}$ (or $\overline{\mathbb{Q}})$, then the pull-back yields an injection

$$
\mathrm{CH}^{*}(X) \otimes \mathbb{Q} \hookrightarrow R\left(X_{\mathbb{C}}\right) \otimes \mathbb{Q} \subset \mathrm{CH}^{*}\left(X_{\mathbb{C}}\right)_{\mathbb{Q}}
$$

To prove the conjecture, it suffices to show that any $K$-rational point $x \in X(K)$ satisfies $[x]=c_{X} \in \mathrm{CH}^{2}\left(X_{\mathbb{C}}\right)$. This would follow from the a priori stronger statement that any $K$-rational point $x \in X(K)$ lies on a rational curve, which is called a 'logical possibility' by Bogomolov [6]. Note that there are other classes of points on K3 surfaces which are known to have fundamental class in $R\left(X_{\mathbb{C}}\right)$, e.g. in [17] this is shown for points that can 
be written as sums of a torsion point on an elliptic curve and a point in the intersection of the elliptic curve with a rational curve.

From the derived point of view, any $K$-rational point $x$ defines a semi-rigid object

$$
k(x) \in \mathrm{D}^{\mathrm{b}}(X),
$$

where $\mathrm{D}^{\mathrm{b}}(X)$ is viewed as a $K$-linear triangulated category. By definition, an object $E \in$ $\mathrm{D}^{\mathrm{b}}(X)$ is called semi-rigid if $\operatorname{Ext}_{X}^{*}(E, E) \simeq H^{*}\left(\mathrm{~S}^{1} \times \mathrm{S}^{1}, K\right)$. For comparison, recall that $E$ was called spherical if $\operatorname{Ext}_{X}^{*}(E, E) \simeq H^{*}\left(\mathrm{~S}^{2}, K\right)$.

The techniques of this article do not allow one to treat semi-rigid objects, but they do show that their simpler spherical cousins behave as expected.

Proposition 5.3. Let $E \in \mathrm{D}^{\mathrm{b}}(X)$ be a spherical object on a smooth projective $K 3$ surface $X$ over a number field $K \subset \mathbb{C}($ or $\overline{\mathbb{Q}})$ such that $\rho\left(X_{\mathbb{C}}\right) \geq 2$. Then under $\mathrm{CH}^{*}(X) \rightarrow$ $\mathrm{CH}^{*}\left(X_{\mathbb{C}}\right)$ its Mukai vector $v^{\mathrm{CH}}(E) \in \mathrm{CH}^{*}(X)$ is mapped to $R\left(X_{\mathbb{C}}\right)$.

Proof. This is an immediate consequence of Corollary 3.3 Indeed, flat base change turns $E$ into a spherical object $E_{\mathbb{C}} \in \mathrm{D}^{\mathrm{b}}\left(X_{\mathbb{C}}\right)$ whose Mukai vector is contained in $R\left(X_{\mathbb{C}}\right)$.

By means of the proposition one can now produce non-trivial classes on $\mathrm{K} 3$ surfaces over number fields that are contained in $R\left(X_{\mathbb{C}}\right)$. In other words, these classes behave as predicted by Conjecture 5.2, but for a less geometric reason than e.g. rational points contained in rational curves.

As it turns out, in fact all spherical objects on the complex $\mathrm{K} 3$ surface $X_{\mathbb{C}}$, which although rigid exist in abundance, are defined over $\overline{\mathbb{Q}}$. This is

Proposition 5.4. Let $X$ be a smooth projective $K 3$ surface over a number field $K \subset \mathbb{C}$. Then any spherical object $F \in \mathrm{D}^{\mathrm{b}}\left(X_{\mathbb{C}}\right)$ is defined over some finite extension $L / K$, i.e. there exists a spherical object $E \in \mathrm{D}^{\mathrm{b}}\left(X_{L}\right)$ such that $E_{\mathbb{C}} \simeq F$.

Proof. This uses a standard argument that roughly says that all points of a zero-dimensional moduli space representing a moduli functor defined over an algebraically closed field are defined over the same field. E.g. any line bundle on $X_{\mathbb{C}}$ is defined over $\overline{\mathbb{Q}}$ (and hence over some finite extension of $K$ ), because the Picard variety for $X_{\overline{\mathbb{Q}}}$ lives over $\overline{\mathbb{Q}}$.

In our case we use Inaba's moduli space of simple complexes (cf. [16] for a more gen-

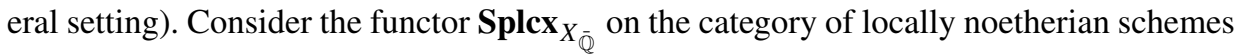
over $\overline{\mathbb{Q}}$, which in particular sends the spectrum of any finitely generated field extension $L / \overline{\mathbb{Q}}$ to the set of isomorphism classes of all bounded complexes $E \in \mathrm{D}^{\mathrm{b}}\left(X_{L}\right)$ with $\operatorname{Ext}_{X_{L}}^{0}(E, E)=L$ and $\operatorname{Ext}_{X_{L}}^{i}(E, E)=0$ for $i<0$. Then it is shown in [13] that the étale sheafification Splcx $X_{\overline{\mathbb{Q}}}^{\text {et }}$ is represented by an algebraic space over $\overline{\mathbb{Q}}$, which we denote $\mathbf{S p l}$.

Any bounded complex $F \in \mathrm{D}^{\mathrm{b}}\left(X_{\mathbb{C}}\right)$ is defined over some finitely generated field extension $L / \overline{\mathbb{Q}}$. Thus, a spherical $F \in \mathrm{D}^{\mathrm{b}}\left(X_{\mathbb{C}}\right)$ can be seen as an $L$-rational point of Spl. The vanishing of $\operatorname{Ext}^{1}(F, F)$ shows that the Zariski tangent space at the corresponding point in $\mathbf{S p l}_{L}$ is trivial. In particular, locally around the point corresponding to $F$ the algebraic space $\mathbf{S p l}$ is zero-dimensional and we may therefore assume it is a scheme over $\overline{\mathbb{Q}}$. 
To conclude, use the following straightforward argument from commutative algebra. Let $A$ be a finitely generated $k$-algebra over an algebraically closed field $k$, let $L / k$ be any extension, and let $B:=A \otimes_{k} L$. Let $\mathbf{n} \subset B$ be a maximal ideal and suppose that $\mathbf{m}:=A \cap \mathbf{n}$ is maximal in $A$. If now $\mathbf{n} / \mathbf{n}^{2}=0$, then $k=A$. Indeed, Nakayama's lemma immediately shows that $B$ must be a field and hence $\mathbf{m}=A \cap \mathbf{n}=0$, i.e. $A$ is a field. Since $A$ is a finitely generated algebra over the algebraically closed field $k$, this yields $A=k$. In order to reduce to the case that $\mathbf{m}$ is maximal, i.e. that the $L$-rational point of Spl is a closed point, take a generic closed point $P$ in the Zariski closure of the image of $\operatorname{Spec}(L) \rightarrow$ Spl. By semi-continuity it will correspond to a spherical object on $X_{k(P)}$. Then the above argument applies and shows that $P$ is isolated and therefore equals the original $L$-rational point.

This shows that any spherical object $F \in \mathrm{D}^{\mathrm{b}}\left(X_{\mathbb{C}}\right)$ is defined eventually over $\overline{\mathbb{Q}}$ and hence over some finite extension of $K$.

The deformation techniques used to prove Proposition 2.5 would allow one to avoid moduli spaces of simple complexes and to work solely with moduli spaces of bundles, but the above proof seems more conceptual.

Remark 5.5. As the reader will have noticed, the proof also shows that the FourierMukai kernel $\mathcal{F}$ of any autoequivalence $\Phi_{\mathcal{F}}: \mathrm{D}^{\mathrm{b}}\left(X_{\mathbb{C}}\right) \stackrel{\sim}{\rightarrow} \mathrm{D}^{\mathrm{b}}\left(X_{\mathbb{C}}\right)$ is defined over $\overline{\mathbb{Q}}$. In other words, $\operatorname{Aut}\left(\mathrm{D}^{\mathrm{b}}\left(X_{\overline{\mathbb{Q}}}\right)\right) \simeq \operatorname{Aut}\left(\mathrm{D}^{\mathrm{b}}\left(X_{\mathbb{C}}\right)\right)$. Of course, the same holds for the set of equivalences between two different K3 surfaces both defined over $\overline{\mathbb{Q}}$.

Acknowledgments. I wish to thank C. Voisin for instructive comments on a first version of the paper and R. Kloosterman for his help with an argument in Section 5. Thanks also to E. Mistretta, M. Penegini, and D. Ploog, who have checked Theorem 2 in explicit examples at an early stage of this work.

This work was supported by the SFB/TR 45 'Periods, Moduli Spaces and Arithmetic of Algebraic Varieties' of the DFG (German Research Foundation).

\section{References}

[1] Arbarello, E., Cornalba, M., Griffiths, P., Harris, J.: Geometry of Algebraic Curves. Grundlehren Math. Wiss. 267, Springer (1985) Zbl 0559.14017 MR 0770932

[2] Barth, W., Hulek, K., Peters, C., Van de Ven, A.: Compact Complex Surfaces. Ergeb. Math. Grenzgeb. 3, Springer (2004) Zbl 1036.14016 MR 2030225

[3] Bloch, S.: Lectures on Algebraic Cycles. Duke Univ. Math. Ser. 4, Duke Univ. (1980) Zbl 0436.14003 MR 0558224

[4] Bloch, S.: Algebraic cycles and values of $L$-functions. J. Reine Angew. Math. 350, 94-108 (1984) Zbl 0527.14008 MR 0743535

[5] Beauville, A., Voisin, C.: On the Chow ring of a K3 surface. J. Algebraic Geom. 13, 417-426 (2004) Zbl 1069.14006 MR 2047674

[6] Bogomolov, F., Tschinkel, Y.: Rational curves and points on K3 surfaces. Amer. J. Math. 127, 825-835 (2005) Zbl 1082.14025 MR 2154371

[7] Bridgeland, T.: Stability conditions on K3 surfaces. Duke Math. J. 141, 241-291 (2008) Zbl 1138.14022 MR 2376815 
[8] Fulton, W.: Intersection Theory. 2nd ed., Ergeb. Math. Grenzgeb. 2, Springer (1998) Zbl 0885.14002 MR 1644323

[9] Hartshorne, R.: Algebraic Geometry. Grad. Texts in Math. 52, Springer (1977) Zbl 0367.14001 MR 0463157

[10] Huybrechts, D.: Fourier-Mukai Transforms in Algebraic Geometry. Oxford Math. Monogr., Oxford Univ. Press (2006) Zbl 1095.14002 MR 2244106

[11] Huybrechts, D., Lehn, M.: The Geometry of Moduli Spaces of Sheaves. Aspects Math. E31, Vieweg, Braunschweig (1997) Zbl 0872.14002 MR 1450870

[12] Huybrechts, D., Macrì, E., Stellari, P.: Derived equivalences of K3 surfaces and orientation. Duke Math. J. 149, 461-507 (2009) Zbl pre05611496 MR 2553878

[13] Inaba, M.: Toward a definition of moduli of complexes of coherent sheaves on a projective scheme. J. Math. Kyoto Univ. 42, 317-329 (2002) Zbl 1063.14013 MR 1966840

[14] Kuleshov, S.: An existence theorem for exceptional bundles on K3 surfaces. Math. USSR Izvestiya 34, 373-388 (1990) Zbl 0706.14009

[15] Lazarsfeld, R.: Brill-Noether-Petri without degenerations. J. Differential Geom. 23, 299-307 (1986) Zbl 0608.14026 MR 0852158

[16] Lieblich, M.: Moduli of complexes on a proper morphism. J. Algebraic Geom. 15, 175-206 (2006) Zbl 1085.14015 MR 2177199

[17] Maclean, C.: Chow groups of surfaces with $h^{2,0} \leq 1$. C. R. Math. Acad. Sci. Paris 338, 55-58 (2004) Zbl 1046.14003 MR 2038085

[18] Mori, S., Mukai, S.: The uniruledness of the moduli space of curves of genus 11. In: Algebraic Geometry (Tokyo/Kyoto, 1982), Lecture Notes in Math. 1016, Springer, 334-353 (1983) Zbl 0557.14015 MR 0726433

[19] Mukai, S.: On the moduli space of bundles on K3 surfaces, I. In: Vector Bundles on Algebraic Varieties, Oxford Univ. Press, 341-413 (1987) Zbl 0674.14023 MR 0893604

[20] Mumford, D.: Rational equivalence of 0-cycles on surfaces. J. Math. Kyoto Univ. 9, 195-204 (1968) Zbl 0184.46603 MR 0249428

[21] Rapoport, M., Schappacher, N., Schneider, P.: Beilinson's Conjectures on Special Values of $L$-functions. Perspectives Math., Academic Press (1988) Zbl 0635.00005 MR 0944994

[22] Saint-Donat, B.: Projective models of K3 surfaces. Amer. J. Math. 96, 602-639 (1974) Zbl 0301.14011 MR 0364263

[23] Seidel, P., Thomas, R.: Braid group actions on derived categories of coherent sheaves. Duke Math. J. 108, 37-108 (2001) Zbl 1092.14025 MR 1831820

[24] Voisin, C.: Théorie de Hodge et géométrie algébrique complexe. Cours spécialisés 10, Soc. Math. France (2002) Zbl 1032.14001 MR 1988456 